\title{
Evaluation Approach for Post-Stroke Rehabilitation via Virtual Reality Aided Motor Training
}

\author{
Shih-Ching Yeh ${ }^{1}$, Jill Stewart ${ }^{2}$, Margaret McLaughlin ${ }^{3}$, Thomas Parsons ${ }^{4}$, Carolee J. Winstein ${ }^{2}$, Albert \\ Rizzo $^{4}$ \\ ${ }^{1}$ Department of Computer Science, ${ }^{2}$ Department of Biokinesiology \&Physical Therapy, ${ }^{3}$ Annenberg School for \\ Communication, ${ }^{4}$ Institute for Creative Technologies \\ University of Southern California, Los Angeles, CA \\ shihchiy@usc.edu, jcstewar@usc.edu, ․mclaugh@usc.edu, TParsons@ict.usc.edu, winstein@usc.edu, \\ arizzo@usc.edu
}

\begin{abstract}
This paper is to introduce an evaluation approach that is applied on clinical data collected from a virtual reality aided motor training for post-stroke rehabilitation. The goal of the proposed evaluation approach is to diagnose patient's current status (performance) and detect patient's change of status (progression). Three kinematics measures, performance time, movement efficiency, and moving speed, are defined to represent kinematics features. 3D performance map and progression map are generated on the base of kinematics measures to visualize the results of patient's behavior. The case study clearly reveals the patient's current status of with respect to his/her range of motion, composed of pitch, yaw and arm length. Further, progression is found and visualized quantitatively over a series of practice sessions.
\end{abstract}

Keywords: Virtual reality, rehabilitation, evaluation approach, human computer interaction

\section{Introduction}

\section{Background}

In the US, more than 700,000 people annually suffer a stroke and this has been cited as one of the leading causes of severe, long-term disability [1]. The disability manifests itself in functional impairments such as difficulty handling multiple activities of daily living, such as dressing, preparing and eating a meal, bathing, etc. As such, quality of life may be severely impacted by stroke [2][3] and more effective methods for rehabilitating lost functioning are a high priority.

Fortunately, loss of upper extremity (UE) function can be recovered or improved via task-oriented motor training which practices activity that targets specific relevant movement, is highly repetitive, and is intensified in a hierarchical fashion based on patient progress. A good motor training task should be designed to target a specific functional deficit (i.e., pointing, grasping, twisting, stretching) and the intensity of exercise should be based on both ongoing status and desired therapeutic goals such as increased movement speed, accuracy, efficiency, distance, orientation, etc. However, motor-training tasks used for conventional therapy are questionable due to limited capacity to systematically control stimulus presentations and to precisely capture motor response performance in real time. Problems with the controlled manipulation of the physical exercise objects limit the ability to vary intensity level in a flexible and dynamic way. The adjustment of environmental parameters in the physical world needed to modulate the intensity of practice can be labor intensive and tedious work for physical therapists and is often deemed to not be cost-effective by third party insurers. Unfortunately this often leads to limited economic support for rehabilitation that could, in the long run, make a real difference. Moreover, data collected from the conventional therapy process is often too limited in type and extent to reliably evaluate the status or performance of the patient. Finally, one possible factor in the mixed outcomes found in rehabilitation research may be in part due to the inability to maintain a patient's motivation and engagement when confronting them with a repetitive series of training challenges. Hence, the integration of gaming features 
in VR-based rehabilitation systems to enhance client motivation is viewed as an important direction to explore. Finally, patient motivation may be minimal when there is little immediate meaningful real time status feedback from the physical environment after a long exercise session.

A Virtual Reality (VR) oriented interactive system provides numerous assets for rehabilitation beyond what is currently available with traditional methods [4][5]. One of the cardinal assets for this form of advanced simulation technology involves the capacity for systematic delivery and control of stimuli. In this regard, an ideal match exists between the stimulus delivery assets of VR simulation approaches and rehabilitation requirements. This "Ultimate Skinner Box" asset can be seen to provide value across the spectrum of rehabilitation approaches, from analysis and training at an analog level targeting component cognitive and physical processes (i.e., selective attention, grip strength, etc.), to the complex orchestration of more complex integrated functional behaviors (e.g., planning, initiating and physically performing the steps required to prepare a meal in a distracting setting). This asset can also be seen to allow for the hierarchical delivery of stimulus challenges across a range of difficulty levels. In this way an individual's rehabilitation can be customized to begin at a stimulus challenge level most attainable and comfortable for them, with gradual progression to higher functional difficulty levels based on the individual's performance. Another primary strength that VR offers rehabilitation is in the creation of simulated realistic environments, within which performance can still be tested and trained in a systematic fashion. By designing virtual environments that not only "look like" the real world, but actually incorporate challenges that require real world functional behaviors, the ecological validity of rehabilitation methods could be enhanced. As well, within a VE, the experimental control required for rigorous scientific analysis and replication can still be maintained within simulated contexts that embody the complex challenges found in naturalistic settings. Thus; VR derived results could have greater predictive validity and clinical relevance for the challenges that clients face in the real world. Further, with the use of advanced sensing systems in VR, a large quantity and wide variety of high quality data can be captured to serve the rehabilitation process. Finally, VRoriented tasks can be equipped with game features that could provide real-time visual, auditory and haptic feedback not only to motivate the patient but also to make the patient feel present within the virtual world. Thus far, early research suggests that the use of VR technology is valuable in improving motor skills for post-stroke rehabilitation on functional deficits including reaching [6], hand function [7] and walking [8] [9].

\section{Previous Work}

We develop a virtual reality aided motor training task: static reaching test, for post-stroke rehabilitation of a functional deficit of the upper extremity. The interactive system is designed both in terms of patientspecific need and from a therapy perspective so as to actively drive the patient's behavior based on the therapist's rehabilitation goal. Within the virtual environment, patient has to reach multiple targets with synchronized forearm and hand movement on their paretic side, shown in Fig. 1. Targets are specified in 3D space with respect to various poses of upper extremity defined by pitch, yaw and extension rate of armlength, shown in Fig. 2.
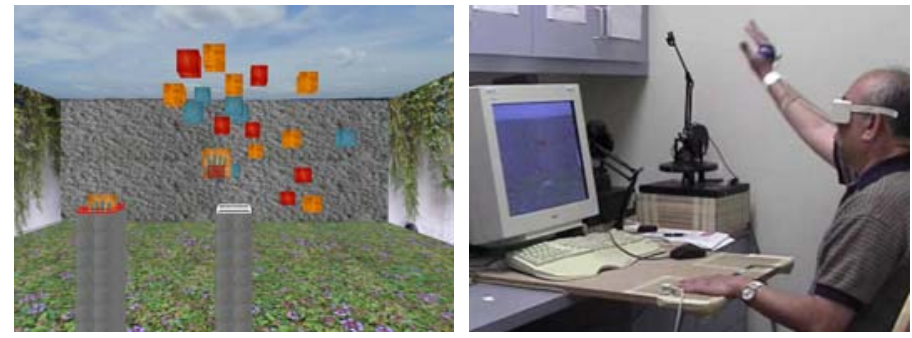

Fig. 1. Virtual reality aided motor training task: static reaching test.

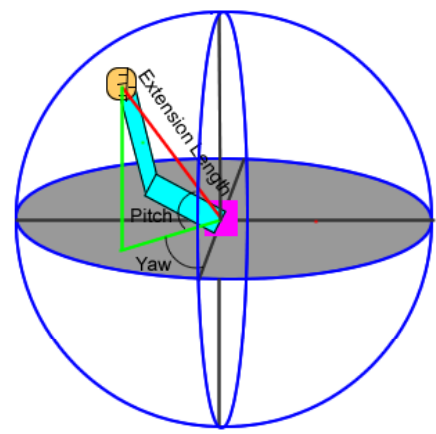

Fig. 2. Pose of upper extremity

A clinical test using this VR task (along with 3 others) was conducted with five post-stroke patients at USC medical school. Patients who volunteer to be subjects have to be screened to see if they meet the inclusion criteria: 1) stroke at least one month prior to the pilot trial; 2) over the age of 18 years; 3) able to attend 12 
training sessions at the Motor Behavior and Neurorehabilitation Laboratory. Subjects are excluded if they have a Mini-Mental Status Exam score below 24, significant limitions in passive range of motion, or no active movement in the hemiparetic UE. Five subjects passed the screening and participated in the test. According to their initial Upper Extremity Fugl-Meyer motor scores, the severity of their disability is classified as severe or moderate, as shown in Table 1.

Table. 1. Severity of disability of subjects

\begin{tabular}{|c|c|c|c|c|c|}
\hline ID & 101 & 102 & 103 & 105 & 106 \\
\hline Severity & Severe & Severe & Moderate & Moderate & Moderate \\
\hline
\end{tabular}

\section{Overview}

One of the challenges in applying such a system to stroke rehabilitation is how can we quantitatively detect the patient's current status or evaluate the patient's progression via the motion data collected? In this paper, we first define three kinematic measures to indicate the performance of patient's motion behavior. Then, we represent the pose of upper extremity with a 2D pitch-yaw grid map. Next, we integrate the kinematic measures with the pitch-yaw map to generate a 3D performance map that can help on the visualization of patient's current status. From it, we develop methodologies to evaluate patient's progression. Finally, case study is made and results are presented.

\section{Evaluation Approach}

\section{Kinematic Measures}

Three types of kinematic measures: performance time, movement efficiency and moving speed, are used to quantitatively evaluate the patient's behavior. They are derived from the continuous position and orientation data of the hand tracker at a data acquisition rate above $60 \mathrm{~Hz}$.

Performance time (PT) is defined as the period between the time when the virtual hand started to move from the start position and the time the virtual hand reached a target in 3D space. It is an index of the moving speed without regard to the moving path. A lower value indicates a higher speed.

Movement efficiency (ME) is defined as the ratio of the actual motion path over the shortest motion path. The shortest motion path is the linear distance between the start position and the position of the virtual target. The actual motion path is the accumulation of linear distance for each time interval through the process of reaching. ME is an index of the patient's moving stability. A lower value of ME indicates a better moving stability.

Moving speed (MS) is defined as the ratio of the actual motion path over performance time. It is an index of moving speed with respect to the motion path.

\section{D Pitch-Yaw Grid Map}

We describe a method to represent the pose of upper extremity, defined by pitch, yaw and arm length, with a series of 2D pitch yaw grid map as below, as shown in Fig. 3. For each specific arm length ratio, the spatial motion zone of the forearm in is as a surface. Such a surface can further be projected onto a plane diagram called 2D pitch-yaw grid map. Each grid on the 2D map represents a certain pose of upper extremity. Also, each grid indicates a certain 3D position in space. 


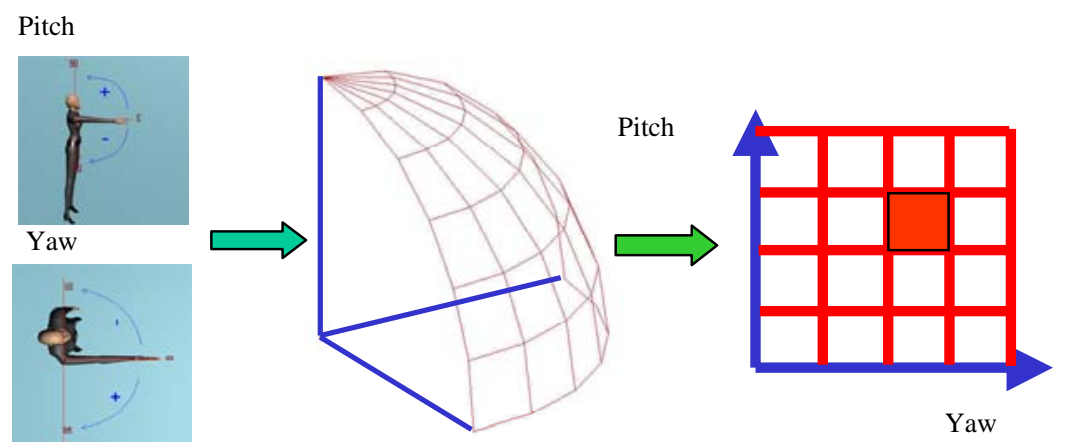

Fig. 3. Represent of the pose of upper extremity: pitch yaw grid map.

\section{Average Chart and Slope Chart}

Average chart and slope charts are built via a two-step classifying process as described below. Data of all test trials (disregard test block) is grouped into multiple datasets where each dataset includes the data belonging to a specific grid on the pitch yaw map. Each dataset is further classified into multiple subdatasets according the time being tested. Kinematic measures are derived for each sub-dataset. Then, in regard with each kinematic measure, average value and slope value of multiple sub-datasets belonging to each dataset are calculated. Further, average value is filled into an average chart with respect to its grid location. As well, slope value is filled into a slope chart. Repeat the same procedures as mentioned above on each grid that an average chart and a slope chart can be filled out. The procedures to develop average chart and slope chart are presented in Fig. 4.

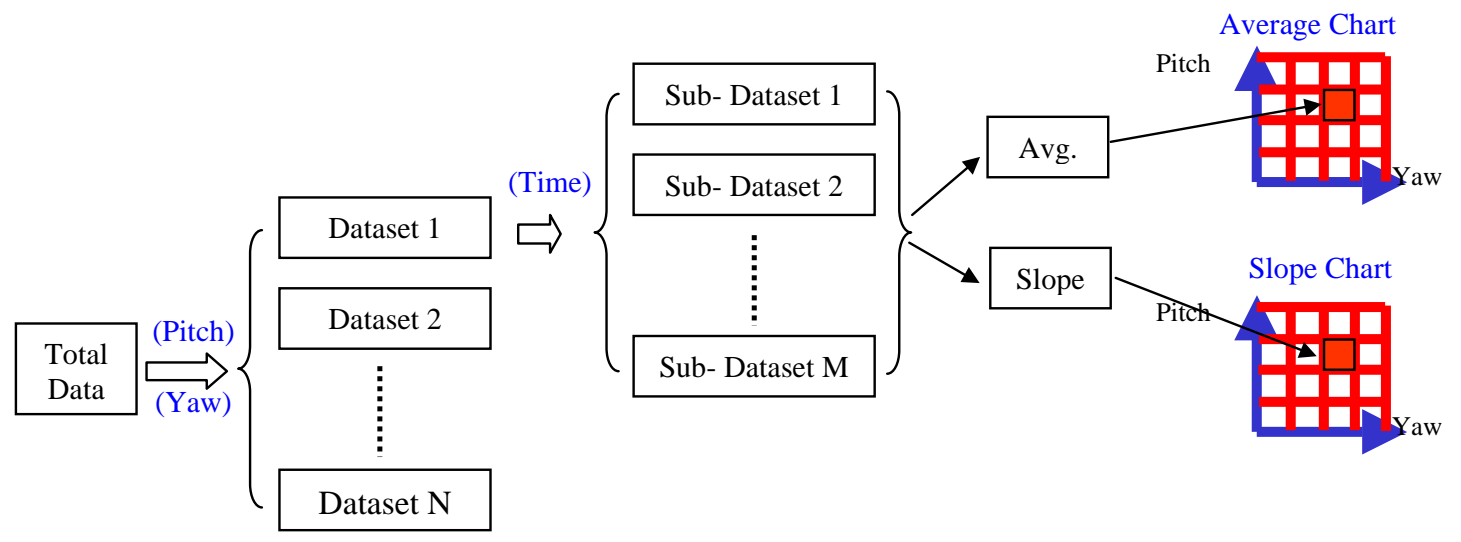

Fig. 4. Development of average chart and slope chart

\section{Performance Map}

3D performance map is built by extruding the average chart with respect to the value of each grid. This can be expressed in Fig. 5. Further, current status can be visualized and be compared from grid to grid via 3D performance map. For PT and ME, the lower the height of bar represents a better performance. For MS, the higher the height of bar represents a better performance. 

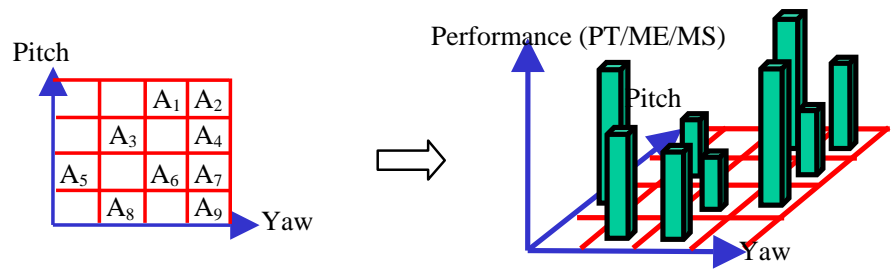

Fig. 5. 3D performance map

\section{Progression Map}

3D progression map is built by extruding the slope chart with respect to the value of each grid. This can be expressed in Fig. 6. A positive slope represents a positive trend on progression for MS but represents a negative trend on progression for PT and ME. To use a positive slope representing a positive trend on progression for three performance measures, the sign of slope is reversed for PT and ME. After the reverse, a negative slope was reset to zero and classified to be no progression. The assumption is that subject can't be worse via the practice. Further, change of status can be visualized and be compared from grid to grid via 3D progression map.
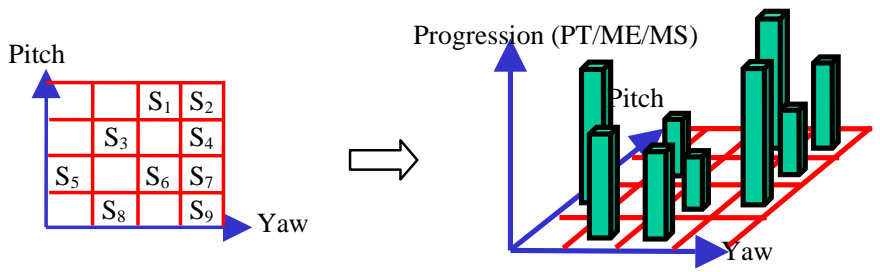

Fig. 6. 3D progression map.

\section{Level Classification}

Both performance and progression are classified into three levels that can be labeled in different colors on the 3D map. Thus, the user can quickly perceive and locate current status or change of status.

Performance is classified into "Excellent”, "Good” and "Fair”. First, all performance data is sorted by its value from better to worse. The average of the first half of the data sets is established as the cutting point between "Excellent" and "Good". Then, the average of the second half of the data sets is established as the cutting point between "Good" and "Fair".

Progression is classified into "Significant", "Minor" and "None”. After the sign reversal, as mentioned above, negative data sets are labeled as "None”. The average of positive sets is established as the cutting point between "Significant” and Minor.

\section{Case Study}

Since a huge amount of data is collected, subject 103 is selected for case study and representative test results.

\section{Visualization of Performance: Arm Length Ratio 60\%}

For each kinematic measure, the performance chart is built via all extracted data sets. The cutting point to classify the status levels is calculated as in Table 2. The 3D performance map is developed as shown in Fig. 
7 and labeled with different colors to indicate the level of current status: red stands for "Excellent", blue stands for "Good" and green stands for "Fair".

At an arm length ratio of $60 \%$, the results show that the subject has a better performance on PT within the zone defined with pitch from 15 to 60 degrees and yaw from -40 to 60 degrees. Poorer performance appears mostly with pitch higher than 60 degrees. With respect to ME, the subject performs better with pitch lower than 75 degrees and yaw lower than 60 degrees. With respect to MS, the subject performs more poorly primarily with pitch higher than 90 degrees or lower than 15 degrees.

(a) PT
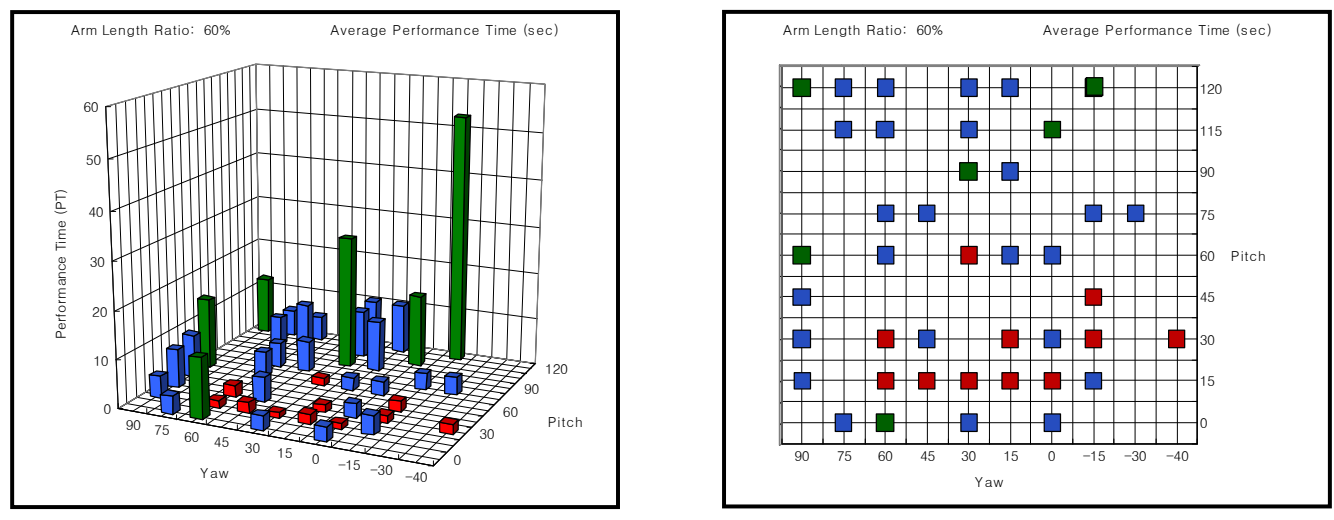

(b) $\mathrm{ME}$
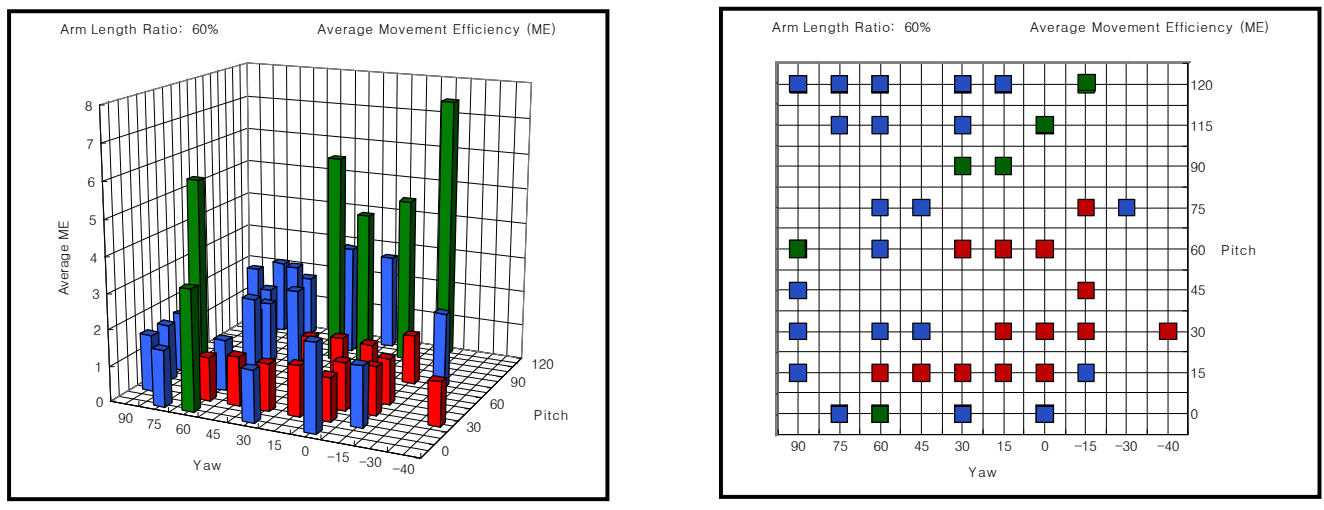

(c) MS
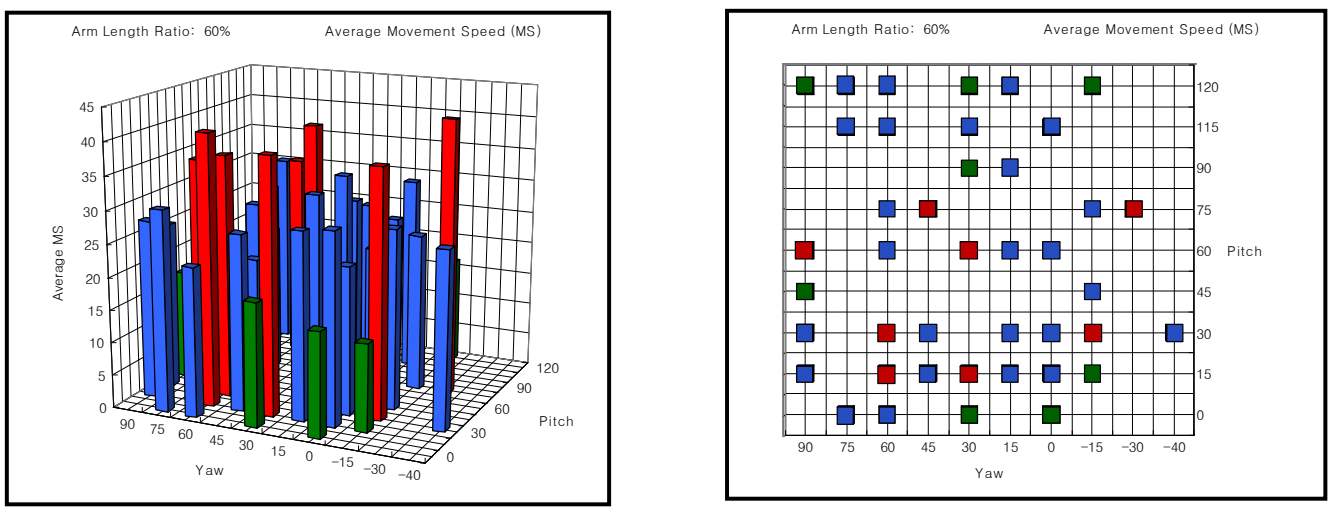

Fig. 7. Performance map of each kinematic measure 
Table. 2. Performance level classification

\begin{tabular}{|c|c|c|c|}
\hline Performance & PT (sec) & ME & MS \\
\hline Excellent & $P T<2.49$ & $M E<1.41$ & $M S \geq 33.42$ \\
\hline Good & $2.49 \leq P T<10.77$ & $1.41 \leq M E<3.24$ & $19.27 \leq M S<33.42$ \\
\hline Fair & $P T \geq 10.77$ & $M E \geq 3.24$ & $M S<19.27$ \\
\hline
\end{tabular}

\section{Visualization of Progression: Arm Length Ratio 60\%}

A progression chart is built for each performance measure. The cutting point for each level of progression was calculated as shown in Table 3. Also, a 3D progression map for each kinematic measure is developed as shown in Fig. 8, with different colors indicating the level of progression, where red stands for "Significant”, blue stands for "Minor" and green stands for "None".

The results indicate that significant progression on PT appears mostly at pitch equal to 120 degrees while no progression appears mostly within the zone defined with pitch lower than 90 degrees and yaw lower than 45 degrees. Significant progression on ME exists in a zone with either pitch higher than 90 degrees or yaw higher than 45 degrees. With regard the MS, significant progress can be seen only in a zone with yaw higher than 60 degrees.

Table. 3. Progression level classification

\begin{tabular}{|c|c|c|c|}
\hline Progression & $-\mathrm{S}_{\mathrm{PT}}$ & $-\mathrm{S}_{\mathrm{ME}}$ & $\mathrm{S}_{\mathrm{MS}}$ \\
\hline Significant & $-S_{P T}>2.46$ & $-S_{M E}>0.56$ & $S_{M S}>5.36$ \\
\hline Minor & $0<-S_{P T} \leq 2.46$ & $0<-S_{M E} \leq 0.56$ & $0<S_{M S} \leq 5.36$ \\
\hline None & $-S_{P T} \leq 0$ & $-S_{M E} \leq 0$ & $S_{M S} \leq 0$ \\
\hline
\end{tabular}

\section{Overview of Performance versus Arm Length Ratio}

The average of each kinematic measure for all zones is calculated for each specific arm length ratio, shown in Fig. 9. It indicates that the PT increases gradually with the increase of arm length ratio. However, ME has no significant change until arm length ratio is equal to 100\%. And after that, ME increases rapidly.

\section{Overview of Progression versus Arm Length Ratio}

At each specific arm length ratio, the percentage of zones with each progression level is calculated, as shown in Fig. 10. At an arm length ratio equal to or higher than $85 \%$, the percentage of zones in progression on PT and ME is obviously higher than for other arm length ratios. However, MS has the highest percentage of zones in progression at two extremes of the arm length ratio: 25\% and $120 \%$. 
(a) PT

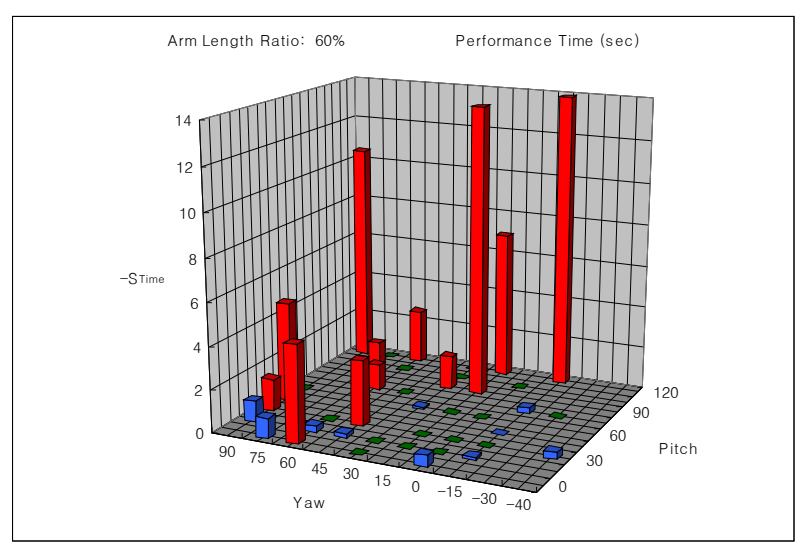

Arm Length Ratio: $60 \%$

Performance Time (sec)

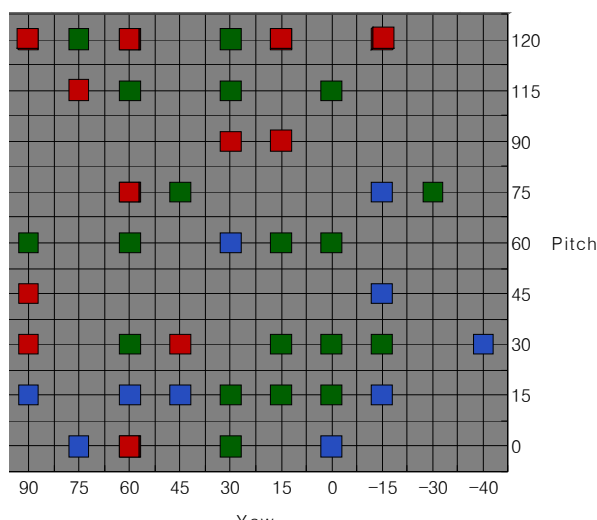

(b) $\mathrm{ME}$
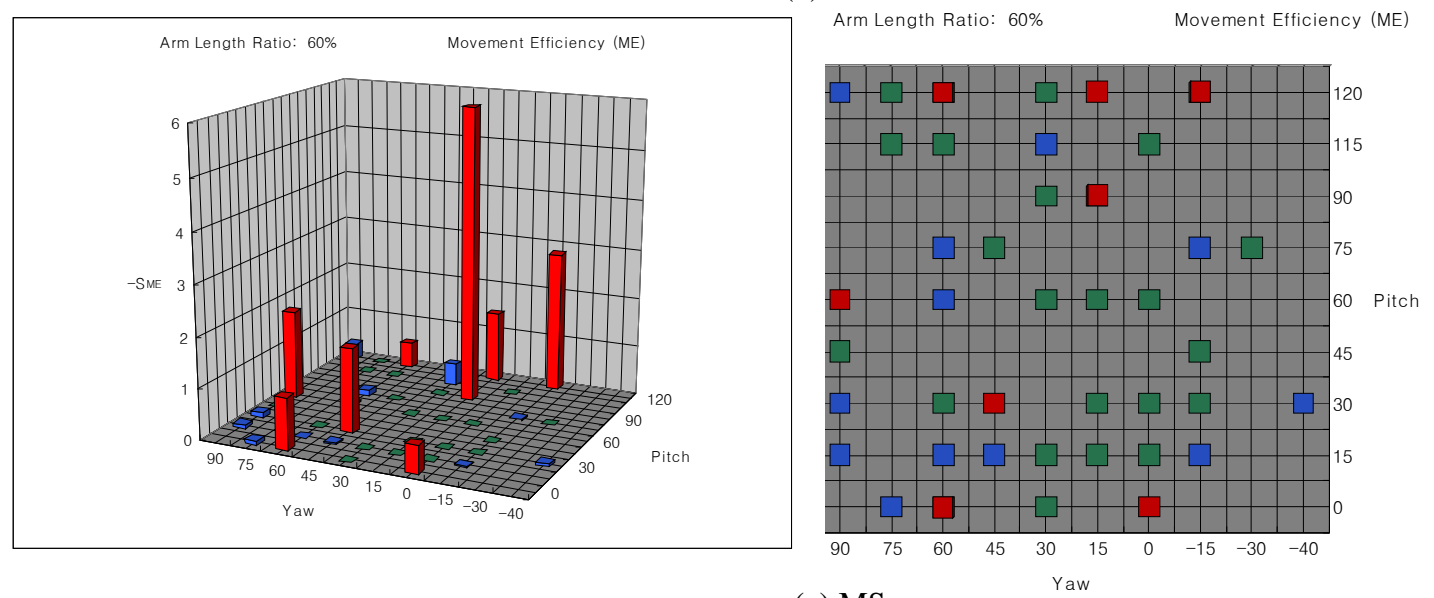

(c) MS

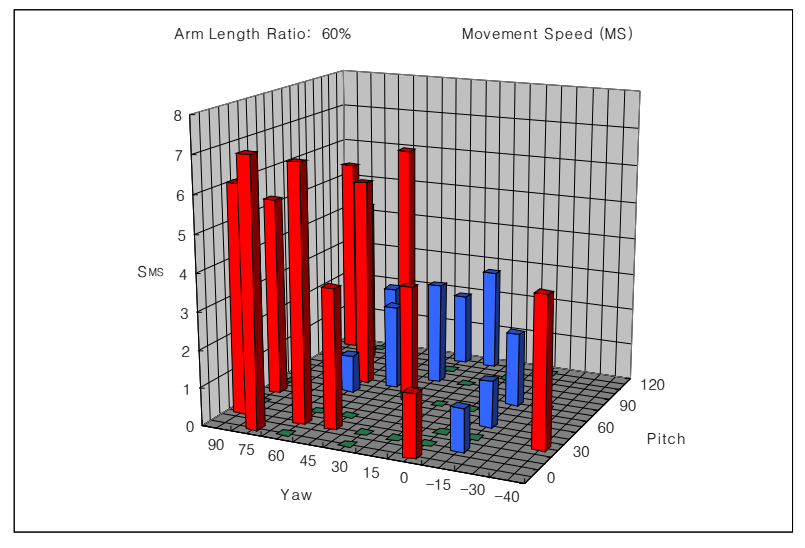

Arm Length Ratio: 60\% Movement Speed (MS)

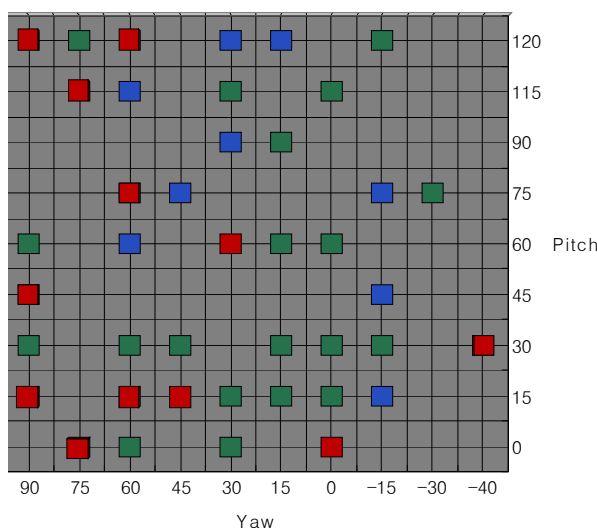

Fig. 8. Progression map of kinematic measures 
(a) PT

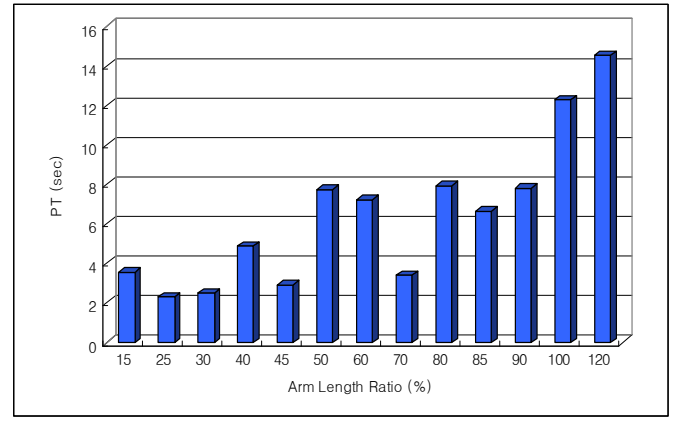

(b) ME

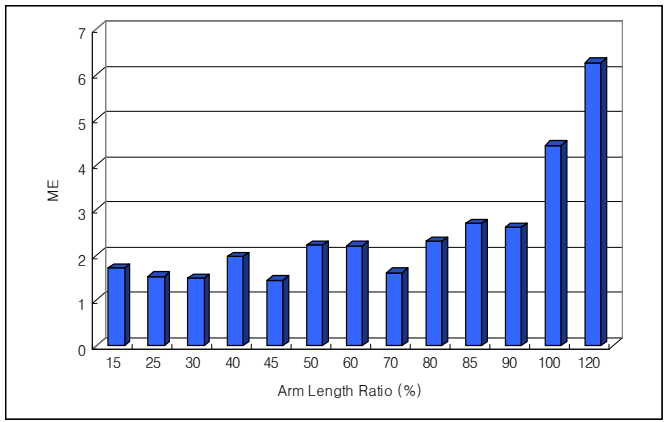

(c) MS

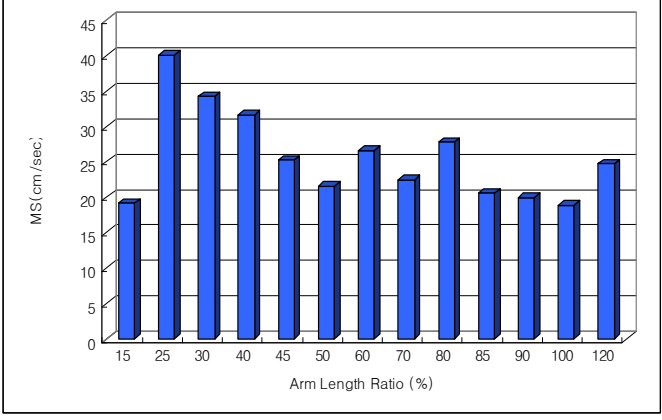

Fig. 9. Performance versus arm length.

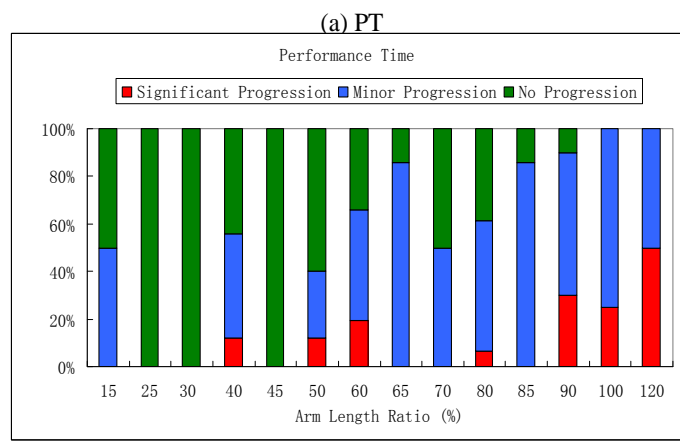

(b) ME

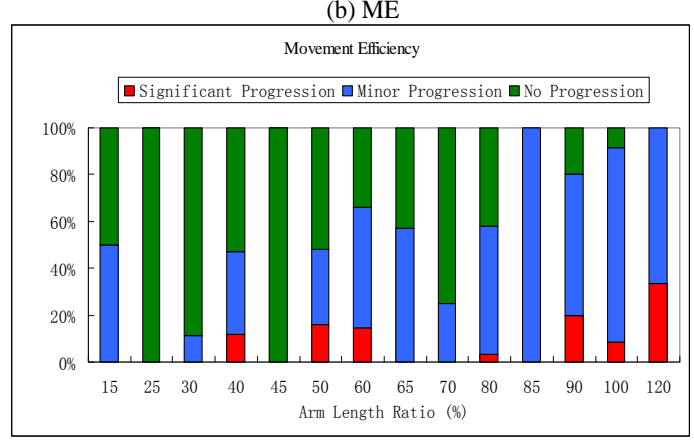

(c) MS

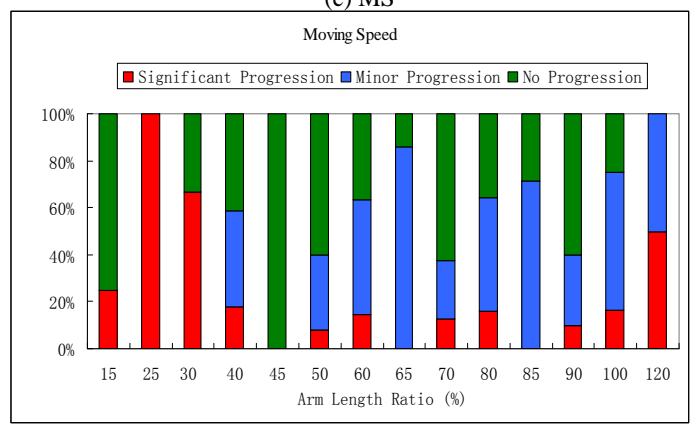

Fig. 10. Performance versus arm length.

\section{Conclusion and Future Work}

\section{Conclusion}

A VR aided upper extremity motor training system has been designed to meet the needs of both patients and therapists. And it has been applied in a clinical pilot test on five stroke patients. Representative kinematics measures, PT, ME and MS are defined to represent kinematics features. Evaluation approach is developed to generate performance map and progression map that can visualize the current status (performance) and the change of status (progression) on the base of kinematics features.

The case study clearly reveals the patient's current status of with respect to his/her range of motion, composed of pitch, yaw and arm length. Further, progression is found and visualized quantitatively over a series of practice sessions. The case study shows that progression appears mostly in zones with lower performance by the patient. 


\section{Future Work}

More patients' data need to be investigated so that statistical model can be further advanced. A larger scale clinical test on more patients and more healthy controls is needed so that more data sets can be collected representatively to develop a complete database for further study. Advanced learning-based algorithms need to be applied so that the huge amount of multiple dimensions can be analyzed systematically. Further, a an easy-to-use tool for automatic diagnosis of current status and evaluation of the patient's progress needs to be developed for use by the clinician.

\section{Acknowledgments}

This research was supported in part by National Institutes of Health Roadmap Initiative grant \# P20 RR20700-01 and by the Integrated Media Systems Center, a National Science Foundation Engineering Research Center, Cooperative Agreement \# EEC-9529152, with additional support from the Annenberg School for Communication, University of Southern California.

\section{Reference}

[1] McLaughlin, M., Rizzo, A., Jung, Y., Peng, W., Yeh, S., Zhu, W., and the USC/UT Consortium for Interdisciplinary Research (accepted for presentation), Haptics-enhanced virtual environments for stroke rehabilitation. Proc. IPSI 2005, Cambridge, MA

[2] Mayo NE, Wood-Dauphinee S, Cote R, Durcan L, Carlton J. Activity, participation, and quality of life 6 months poststroke. Arch Phys Med Rehabil. 2002;83:1035-1042.

[3] Jonsson AC, Lindgren I, Hallstrom B, Norrving B, Lindgren A. Determinants of quality of life in stroke survivors and their informal caregivers. Stroke. 2005;36:803-808.

[4] Holden MK. Virtual environments for motor rehabilitation: review. Cyberpsychol Behav. 2005:8 (3):187-211.

[5] Weiss PL, Katz N. The potential of virtual reality for rehabilitation. J Rehabil Res Dev. 2004;41 (5):vii-x.

[6] Holden M, Todorov E, Callahan J, Bizzi E. Virtual environment training improves motor performance in two patients with stroke: case report. Neurol Rep. 1999;23(2):57-67.

[7] Merians AS, Jack D, Boian R, Tremaine M, Burdea GC, Adamovich SV, Recce M, Poizner H. Virtual realityaugmented rehabilitation for patients following stroke. Phys Ther. 2002;82:898-915.

[8] You SH, Jang SH, Kim YH, Hallett M, Ahn SH, Kwon YH, Kim JH, Lee Y. Virtual reality-induced cortical reorganization and associated locomotor recovery in chronic stroke. An experimenter-blind randomized study. Stroke. 2005;36:1166-1171.

[9] Deutsch JE, Latonio J, Burdea G, Boian R. Post-stroke rehabilitation with the Rutgers Ankle System: a case study. Presence. 2001;10(4):416-430. 